\title{
Transhyphal Electrical Currents in Fungi
}

\author{
By NEIL A. R. GOW \\ Department of Microbiology, University of Aberdeen, Marischal College, \\ Aberdeen $A B 9$ IAS, UK
}

(Received 21 May 1984)

\begin{abstract}
Representative mycelial fungi from the phycomycete, ascomycete and basidiomycete groups (Achlya bisexualis, Neurospora crassa, Aspergillus nidulans, Schizophyllum commune and Coprinus cinereus) all generated steady electrical currents around their hyphal tips; the generation of a transhyphal ion current may therefore be a universal characteristic of hyphal growth. As with all other tip growing organisms, positive current always entered apically and left distally; nongrowing hyphae did not drive transcellular currents. The current density, measured approximately $30 \mu \mathrm{m}$ from the membrane surface at the hyphal tips, varied between 0.05 and $0.60 \mu \mathrm{A} \mathrm{cm}^{-2}$ in different fungi and tended to be larger in wider, rapidly extending hyphae than in thinner, slow growing hyphae. The possibility that these currents serve to localize growth at the apex is discussed.
\end{abstract}

\section{INTRODUCTION}

It has been known for about 60 years that active extension of the fungal hypha is restricted to a region only a few microns long at the very apex (Smith, 1923). However, only recently has there been some understanding of how polarized growth is actually achieved. A combination of ultrastructural, microautoradiographic and cell fractionation studies have been used to show how wall synthesizing enzymes and precursors are initially packaged into vesicular structures behind the tip, then are transferred to the apex by some unknown mechanism. The vesicles then fuse with the apical plasmalemma thereby supplying both new membrane surface and the biosynthetic requirements for tip growth (see review by Gooday, 1983). Perhaps the least understood aspect of this chain of events is the nature of the driving force which translocates and guides the membrane vesicles to the hyphal apex. In this regard, recent reports describing the presence of transcellular electrical currents flowing longitudinally along the lengths of tip growing organisms are particularly significant (see review by Nuccitelli, 1983). Might a bioelectric current be operating in directing the movements of vesicles in hyphal growth?

Jaffe \& Nuccitelli (1974) developed the vibrating probe which made measurement of these weak transcellular electrical currents possible. Jaffe and his collaborators were also the first to demonstrate that these currents are intimately associated with the polarity of cell growth and development (Jaffe, 1979, 1981; Jaffe \& Nuccitelli, 1977). Transcellular electrical currents have now been reported in such a diversity of eukaryotic organisms that they may well be ubiquitous features of the organization of higher cells (Nuccitelli, 1983). The tip growing organisms that have been found to drive transcellular currents include germinating algal eggs (Nuccitelli \& Jaffe, 1974), plant roots (Weisenseel et al., 1979), lily pollen grains (Weisenseel et al., 1975) and the hyphae of water moulds (Armbruster \& Weisenseel, 1983; Kropf et al., 1983, 1984). In each of these systems the current is orientated in the same axis of polarity as the cells, with positive current entering the growing apex and leaving further back. Moreover, the future sites of outgrowth of hyphal branches of Achlya bisexualis (Kropf et al., 1983), the rhizoid of Pelvetia zygotes (Nuccitelli, 1978) and the germ tubes of lily pollen grains (Weisenseel et al., 1975) are preceded and predicted by a localized influx of electrical current, suggesting that the generation 
of a transcellular current is a very early event in the establishment of cell polarity. In $A c$. bisexualis the electrical current has been ascribed to a flux of protons (Kropf et al., 1984), while in Pelvetia and lily pollen grains the inward current is carried in part by calcium and potassium ions, respectively (Nuccitelli, 1978, Weisenseel \& Jaffe, 1976).

Studies of transcellular ion currents may provide new insights into the mechanisms of apical growth of fungi. However, before assuming that the generation of a transhyphal electrical current is a characteristic feature of hyphal growth and therefore worthy of close attention, it must first be established that the phenomenon is widespread amongst mycelial fungi. This paper shows that representative fungi from each of the major taxonomic sub-divisions all drive transhyphal electrical currents. The possible significance of these currents to hyphal growth is discussed.

\section{METHODS}

Organisms and media. Achlya bisexualis female strain T5 was from the American Type Culture Collection. Neurospora crassa RL21a, Aspergillus nidulans G00, and Schizophyllum commune $699 \times 845$ were gifts from C. L. Slayman (University of Yale, Conn., USA), J. Clutterbuck (University of Glasgow, UK) and J. G. H. Wessels (University of Groningen, The Netherlands), respectively. Strains of Mucor mucedo Z43 (+) and Coprinus cinereus H5 $\times$ C692 were donated by G. W. Gooday (this department) and were from the University of Aberdeen Culture Collection.

When using the vibrating probe, it was an advantage to use media of high resistivity (1000 $\Omega \mathrm{cm}$ or more) since the signal voltage increased with increasing medium resistivity. Media of high resistivity contain low ion concentrations. DMA, a defined medium used in the cultivation of Ac. bisexualis, was specially designed to have a high resistivity; its compcsition is described by Kropf et al. (1984). Vogel's minimal medium (Vogel, 1956) and Pontecorvo's minimal medium (Pontecorvo, 1952), used to grow N. crassa and As. nidulans respectively, contain high salts; these media were therefore used at one tenth the stated salt concentration but with all other components (sugars, vitamins, etc.) at normal concentrations. Media with diluted salts were osmotically balanced with mannitol to their original osmolarities. C. cinereus and $S$. commune were grown in media containing $2 \%(\mathrm{w} / \mathrm{v})$ glucose, $0.5 \%$ mycological peptone, $1 \%$ (w/v) malt extract and $0.4 \%$ yeast extract and $M$. mucedo in $2 \%$ glucose, $0.5 \%$ mycological peptone and $2 \%$ malt extract. All media had resistivities of $900-1350 \Omega \mathrm{cm}$ and were at $\mathrm{pH} 6.5$, except for the modified Vogel's medium (pH 6.0). The fungi were grown at $25^{\circ} \mathrm{C}$.

Growth of fungi and measurements of extracellular electric currents. Growth and measurement of electrical currents of $A c$. bisexualis was as described by Gow et al. (1984). Techniques for $N$. crassa, As. nidulans and $M$. mucedo were as follows. Mycelial colonies were grown on thin agar ( $2 \mathrm{~mm}$ depth) in Petri dishes. Oblong blocks of agar 5-7 $\times 15-20 \mathrm{~mm}$ were cut beyond and around the leading edges of colonies so that a sector of undisturbed mycelium was removed with the underlying agar. This block was placed eccentrically in a $5 \mathrm{~cm}$ culture dish so that the leading edge of the mycelium faced the centre of the dish. A no. 1 coverslip, broken to match the size of the agar block, was carefully placed over the colony so that subsequent growth of the hyphae occurred in a flat focal plane. The coverslip/agar block arrangement was anchored to the bottom of the culture dish with a network of strands of epoxy resin (382 Medical Grade Elastomer, Dow Corning, Michigan, USA) which was allowed to harden before the plates were flooded with growth medium. The plates were incubated at $25^{\circ} \mathrm{C}$ until the hyphae grew horizontally away from the edge of agar block into the liquid medium. Where necessary, the mycelial margin was pruned with fine forceps to leave a few well-separated hyphae for examination and measurement of current.

Hyphae of $S$. commune and $C$. cinereus did not grow well using the above method and were therefore prepared in a different way. Agar discs cut from the margins of mycelial colonies were incubated in shake culture until pellets of diameter $15-20 \mathrm{~mm}$ were formed. One pellet was then gently transferred in a volume of the growth medium into a $5 \mathrm{~cm}$ culture dish then left for 2-3 h to recover from physical shock. Once the hyphae at the margin of the colony resumed growth, a large proportion of the mycelial mass was cleared away to leave a few well-separated hyphae as above.

A vibrating probe was used to map extracellular current flow along those hyphae which had grown away from the agar block or mycelial pellet into the liquid medium. Descriptions of the vibrating probe and the application of this instrument to the study of fungal hyphae are given in Jaffe \& Nuccitelli (1974) and Kropf et al. (1984), respectively. During the experiments, specimens and probe were observed at $100 \times$ magnification with an inverted microscope.

\section{RESULTS}

Examples of profiles of electrical current along hyphae of Ac. bisexualis (an aquatic phycomycete), M. mucedo (a terrestrial phycomycete), $N$. crassa and As. nidulans (two 

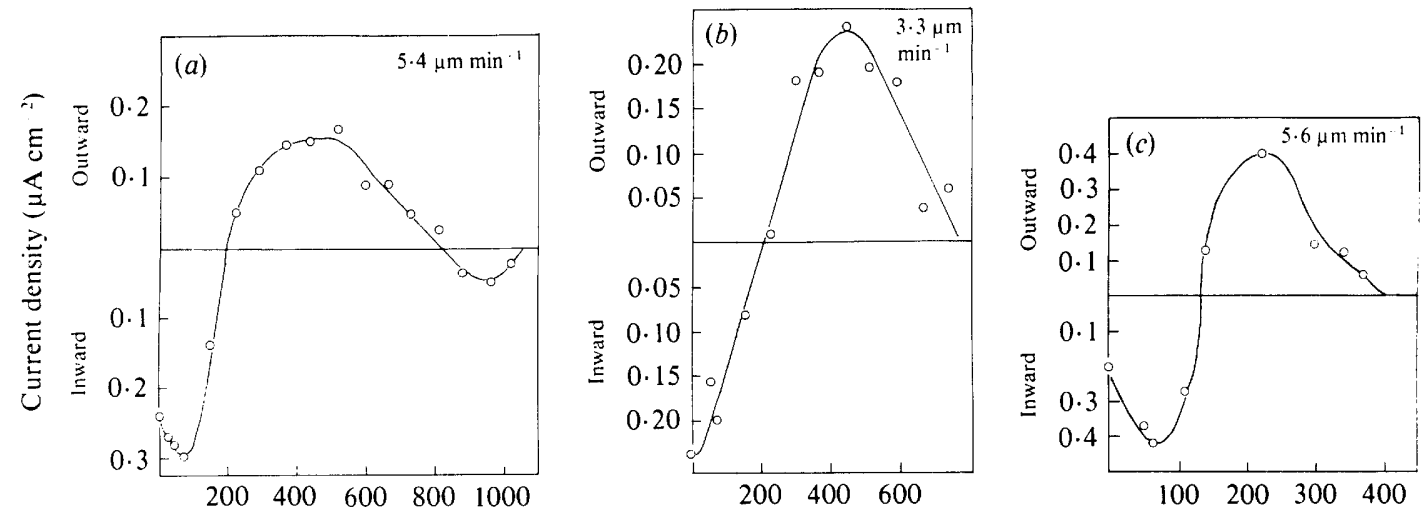

Distance behind apex $(\mu \mathrm{m})$
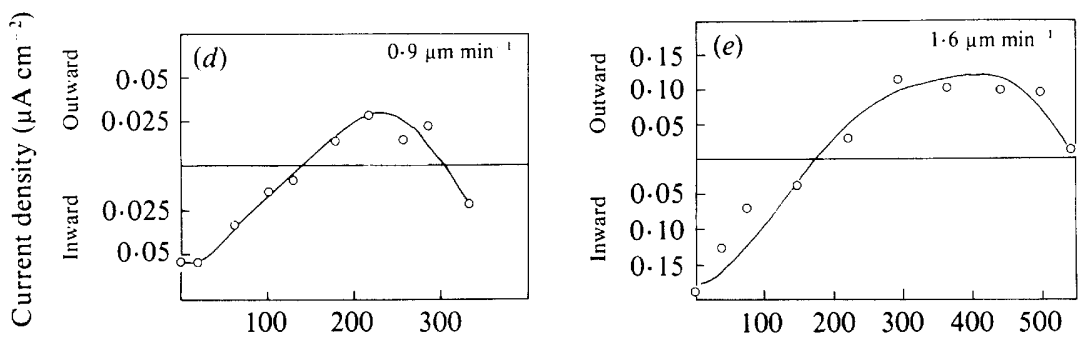

Distance behind apex $(\mu \mathrm{m})$

Fig. 1. Profiles of electrical current flow around hyphae of (a) Ac. bisexualis, (b) M. mucedo, (c) N. crassa, (d) $S$. commune and $(e)$ As. nidulans, growing at the stated extension rates.

ascomycetes) and $S$. commune (a basidiomycete) are given in Fig. $1(a, b, c, e, d$, respectively). Traces of chart recordings showing deflections indicating inward current at hyphal tips are shown for $S$. commune (Fig. $2 a$ ) and $C$. cinereus (Fig. $2 b$ ). A general scheme showing the pattern of electrical current flow around fungal hyphae is shown in Fig. 3. The following characteristics of the electrical currents in these fungi were noted. (i) The six fungi tested, comprising representatives from all the major taxonomic ranks, all generated electrical currents. (ii) Non-growing hyphae did not drive currents (data not shown). (iii) The direction of flow of electrical (positive) current was always inward at the hyphal tip and outward further back. (iv) The total amount of inward current approximately equalled that of the net outward current (an imbalance would cause massive depolarization or hyperpolarization of the membrane). In some cases however, net outward current of a profile was in excess of net inward current (e.g. Fig. 1b): this may relate to the presence of unmeasured branch tips which occurred within the lengths of some of these profiles. $(v)$ For the six fungi examined, the magnitude of the inward current varied from less than $0.05 \mu \mathrm{A} \mathrm{cm}^{-2}$ to $0.6 \mu \mathrm{A} \mathrm{cm} \mathrm{cm}^{-2}$, with the largest current densities tending to correlate with those hyphae which were of wide diameter and rapid growth rate. However, no simple relationship between growth rate and current density was found in these experiments. Although measurements were made as close as possible to the hyphae $(\sim 30 \mu \mathrm{m})$, the current density at the membrane surface may be considerably larger than these values. The magnitude of the current of $C$. cinereus was close to the detection limit of the vibrating probe. Indeed, the transcellular currents of some hyphae of this organism were often so small as to be difficult to differentiate unequivocally the signal from background noise. 
(a)

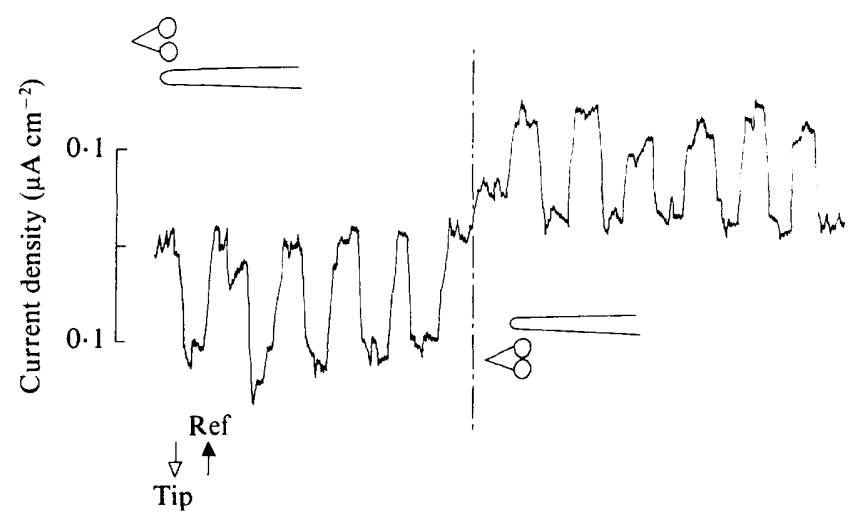

(b)

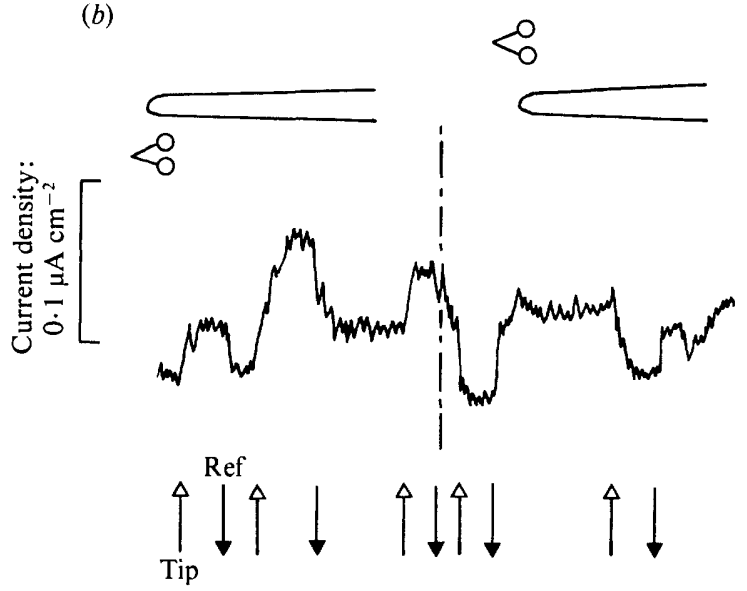

Fig. 2. Tracing of chart recordings showing inward current of the hyphal tips of $(a) S$. commune and $(b)$ $C$. cinereus. The probe was moved successively from a position closely adjacent to the hyphal tip then to a reference position away from the tip as indicated by the arrows. The position of the probe relative to the hyphae is shown; all deflections correspond to inwardly directed electric current. The extension rates of the hyphae were $(a) 0.95 \mu \mathrm{m} \mathrm{min}^{-1}$ and $(b) 0.5 \mu \mathrm{m} \mathrm{min}{ }^{-1}$.

\section{DISCUSSION}

Slayman \& Slayman (1962) used intracellular microelectrodes to measure the membrane potential at the hyphal apex and at distances behind the tip of $N$. crassa. They found the potential at the tip to be as much as $100 \mathrm{mV}$ less negative than distally. This result indicated that ion transport systems may not be homogeneously distributed along these hyphae and that there would be a net flow of positive charges along the hyphae as a consequence of the longitudinal potential difference. The results presented here, measuring extracellular current flow around hyphae of $N$. crassa, confirm this report. Other taxonomically diverse mycelial fungi also translocated positive charges from the tip to the distal hypha. Of the six species examined only Ac. bisexualis had been shown previously to generate a transcellular ion current (Kropf et al., 1983, 1984). Hyphae and sporangia of the water moulds of Achlya debaryana (Armbruster \& Weisenseel, 1983) and Blastocladiella emersonii (Stump et al., 1980) can also be added to the list of fungi which have now been shown to possess currents. This phenomenon would therefore seem to be widespread in the fungi; the question that arises concerns the possible functions of these currents in fungal growth.

Jaffe and others have postulated that the flow of electrical current may confer polarity by establishing intracellular electrophoretic fields which could move and organize appropriately 


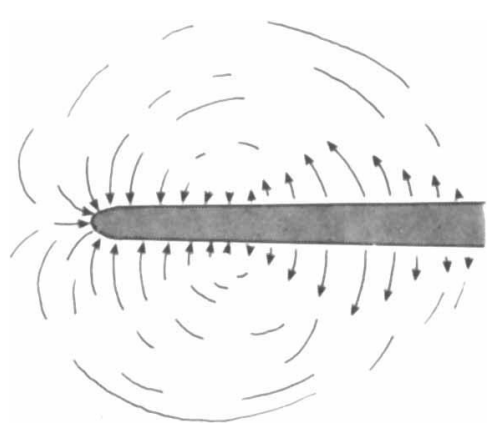

Fig. 3. Pattern of transhyphal ion/electric current around fungal hyphae.

charged molecules in the cytoplasm or plasma membrane (Jaffe, 1977; Jaffe et al., 1974; Quatrano, 1978). The simplicity of such a mechanism is very appealing and a variety of recent theoretical and experimental reports (mainly concerning bioelectric currents in animal cells) strongly support this hypothesis (see review by Nuccitelli, 1983). It is tempting, therefore, to speculate that an intracellular electric field in fungal hyphae may cause electrophoresis of membrane vesicles laterally to the hyphal tip, thereby polarizing growth. The observation that non-growing hyphae failed to circulate current would support this notion.

However, Kropf et al. (1983) have presented evidence which indicates that such a mechanism is unlikely to account for growth localization at hyphal tips of $A c$. bisexualis. They showed that when Achlya branches, the inward current at the parent hyphal tip is often affected transitorily, becoming diminished and sometimes reversed in direction (Kropf et al., 1983). Even when the direction of the current was reversed, these hyphae continued to extend at the same rate. Thus if a self-electrophoretic mechanism was operating, the vesicles would be expected, under these conditions, to be moving away from the growing apex.

Further work in Achlya showed that the electrical current was probably carried by protons (Kropf et al., 1984) and that protons (as opposed to charge) flowed into parent hyphal tips even when hyphae branched (Gow et al., 1984). The current-reversal phenomenon may therefore be due to a transitory flux of some other ion which masked the continued apical influx of protons (Kropf et al., 1984; Gow et al., 1984). In Achlya at least, it would therefore appear that apical growth correlates with the driving of a transcellular proton flux but not with the flow of electrical current per se. If self-electrophoresis cannot always provide an adequate explanation for growth localization, how else might transcellular ion currents be involved in the regulation of cell polarity? One possibility is that the ionic species rather than the electrical current is of importance. In Achlya, for instance, the apical cytosol may be locally acidic, since protons leak into hyphal tips (Gow et al., 1984; Kropf et al., 1984). Local pH may affect and regulate vesicularcytoskeleton interactions which in turn may regulate vesicle translocation and localization (Howard, 1981; Harold, 1982; Gooday, 1983). The cytoskeletal arrangement of cells can also be influenced by electric fields (Luther et al., 1983) thus it could be argued that electrical current may regulate cell polarity through direct interactions with the cytoskeleton.

Another possibility which must be regarded seriously is that the association of current flow and apical growth may be of no morphogenetic significance. Ion transport systems may be segregated for reasons other than the necessity to drive transcellular ion currents. In $A c$. bisexualis the proton circulation seems to correspond to a spatially extended chemiosmotic system (Kropf et al., 1984; Gow et al., 1984). Inward current is probably due to the localization of amino acid/proton symporters at the hyphal tip. Since these hyphae are also chemotropic towards amino acids (Musgrave et al., 1977; R. Harold, unpublished) it could be argued that the transcellular electrical current arises as an inconsequential result of the necessity to localize a nutrient uptake mechanism at the growing apex. However, the seemingly universal correlation of current generation with polarized growth in very different organisms and the reports of currents that precede and predict locations of future growth strongly suggest the association between transcellular ion current and polarized growth is more than coincidence. Future studies 
on transhyphal ion currents may therefore provide us with new insights into how fungi, and eukaryotic cells in general, polarize and localize growth.

Since this paper was accepted for publication, Horwitz et al. (1984) have described the presence of an electrical current entering the hyphal tips of Trichoderma harzianum (a deuteromycete).

I thank Franklin Harold and John Caldwell in whose laboratories this work was carried out, and Clifford Slayman, J. G. H. Wessels, John Clutterbuck and Graham Gooday for gifts of fungal cultures. Technical assistance from Diane Nicholl is gratefully acknowledged. This research was supported by Public Health Service Grant AI-03568 from the National Institute of Allergy and Infectious Diseases and National Science Foundation Grant PCM 8009439 from the Metabolic Biology Program.

\section{REFERENCES}

Armbruster, B. L. \& Weisenseel, M. H. (1983). Ionic currents traverse growing hyphae and sporangia of the mycelial water mould Achlya debaryana. Protoplasma 115, 65-69.

GoOdAY, G. W. (1983). The hyphal tip. In Fungal Differentiation: a Contemporary Synthesis, pp. 315356. Edited by J. E. Smith. New York: Marcel Dekker.

Gow, N. A. R., Kropf, D. L. \& Harold, F. M. (1984). Growing hyphae of Achlya bisexualis generate a longitudinal $\mathrm{pH}$ gradient in the surrounding medium. Journal of General Microbiology 130, 29672974.

HAROLD, F. M. (1982). Pumps and currents: A biological perspective. Current Topics in Membranes and Transport 16, 485-516.

Horwitz, B. A., Weisenseel, M. M., Dorn, A. \& GRESSEL, J. (1984). Electric currents around growing trichoderma hyphae, before and after autoinduction of conidiation. Plant Physiology 74, 912-916.

HowARD, R. J. (1981). Ultrastructural analysis of hyphal tip cell growth in fungi: Spitzenkorper, cytoskeleton and endomembranes after freeze-substitution. Journal of Cell Science 48, 89-103.

JAFFE, L. F. (1977). Electrophoresis along cell membranes. Nature, London 265, 600-602.

JAFFE, L. F. (1979). Control of development by ionic currents. In Membrane Transduction Mechanisris. Society for General Physiologists, vol. 33, pp. 199231. Edited by R. A. Cone \& J. E. Dowling. New York: Raven Press.

JAFFE, L. F. (1981). The role of ionic currents in establishing developmental pattern. Philosophical Transactions of the Royal Society B295, 553566.

JAFFE, L. F. \& Nuccitelli, R. (1974). An ultrasensitive vibrating probe for measuring steady extracellular currents. Journal of Cell Biology 63, 614628.

JAFFE, L. F. \& Nuccitelli, R. (1977). Electrical controls of development. Annual Review's of Biophysics and Bioengineering 6, 445-476.

JAFFe, L. F., Robinson, K. R. \& Nuccitelli, R. (1974). Local cation entry and self-electrophoresis as an intracellular localization mechanism. Annals of the New York Academy of Sciences 238, 372-389.

Kropf, D. K., Lupa, M. D., Caldwell, J. C. \& HAROLD, F. M. (1983). Cell polarity: endogenous ion currents precede and predict branching in the water mould Achlya. Science 220, 1385-1387.
Kropf, D. K., Caldwell, J. C., Gow, N. A. R. \& HAROLD, F. M. (1984). Transcellular ion currents in the water mould Achlya. Amino acid/proton symport as a mechanism of current entry. Journal of Cell Biology 99, 486-496.

Luther, P. W., Peng, H. B. \& Lin, J. J. C. (1983). Changes in cell shape and actin distribution induced by constant electric fields. Nature, London 303, 6164.

Musgrave, A., Ero, L., Scheffer, R. \& Demlers, E. (1977). Chemotropism of Achlya bisexualis germ hyphae to casein hydrolysate and amino acids. Journal of General Microbiology 101, 65-70.

NuCCITELLI, R. (1978). Öoplasmic segregation and secretion in the Pelvetia egg is accompanied by a membrane generated electrical current. Developmental Biology 62, 13-33.

NuCCitelli, R. (1983). Transcellular ion currents. Signals and effectors of cell polarity. Modern Cell Biology 2, 451--481.

NuCCitelli, R. \& JAFFe, L. F. (1974). Spontaneous current pulses through developing fucoid eggs. Proceedings of the National Academy of Sciences of the United States of America 71, 4855-4859.

Pontecorvo, G. (1952). The genetics of Aspergillus nidulans. Advances in Genetics 5, 141-238.

Quatrano, R. (1978). Development of cell polarity. Annual Reviews in Plant Physiology 29, 487-510.

Slayman, C. L. \& Slayman, C. W. (1962). Measurement of membrane potentials in Neurospora. Science 136, 876-877.

Smith, J. H. (1923). On the apical growth of fungal hyphae. Annals of Botany (London) 37, 341-343.

StUmp, R. F., Robinson, K. R., HaRold, R. L. \& HaRold, F. M. (1980). Endogenous electrical currents in the water mould Blastocladiella emersonii during growth and sporulation. Proceedings of the National Academy of Sciences of the United States of America 77, 6673-6677.

Vogel, H. J. (1956). A convenient growth medium for Neurospora. Microbial Genetics Bulletin 13, 42-44.

Weisenseel, M. M. \& JAFFE, L. F. (1976). The major growth current through lily pollen tubes enters as $\mathrm{K}^{+}$ and leaves as $\mathrm{H}^{+}$. Planta $133,1-7$.

Weisenseel, M. M., Nuccitelli, R. \& Jaffe, L. F. (1975). Large electrical currents traverse growing pollen tubes. Journal of Cell Biology 66, 556-567.

Weisenseel, M. M., Dorn, A. \& JAFFe, L. F. (1979). $\mathrm{Natural} \mathrm{H}^{+}$currents traverse growing roots of barley (Hordeum vulgare L.). Plant Physiology 64, 512-518. 